\title{
ESTUDO COMPARATIVO DE DIFERENTES TÉCNICAS DE AVALIAÇÃO DA CONCENTRAÇÃO ESPERMÁTICA EM SUÍNOS
}

\author{
COMPARATIVE STUDY OF DIFFERENT TECHNIQUES OF EVALUATION \\ OF SPERMATIC CONCENTRATION IN SWINES
}

\author{
Murgas, L.D.S. ${ }^{1}$, Lima, D. ${ }^{1}$, Alvarenga, A.L.N. ${ }^{1}$, Zangeronimo, M.G. ${ }^{1}$, Oberlender, G. ${ }^{1 *}$ \\ e Oliveira, S.L. ${ }^{2}$
}

\begin{abstract}
${ }^{1}$ Departamento de Medicina Veterinária. Universidade Federal de Lavras (UFLA/MG). CEP 37200-000. Lavras. Minas Gerais. Brasil. *guilherme_oberlender@yahoo.com.br ${ }^{2}$ Departamento de Ciências Biológicas. Universidade Federal de Goiás. Jataí, Goiás. Brasil.
\end{abstract}

\section{PaLAVRAS CHAVE ADICIONAIS}

Espectrofotômetro. Espermiodensímetro. Câmara de Neubauer.

\section{RESUMO}

A determinação precisa e acurada da concentração espermática do ejaculado suíno é importante na produção de doses inseminantes com um número determinado de espermatozóides. O objetivo do trabalho foi comparar a eficiência de algumas técnicas de mensuração da concentração espermática no sêmen e determinar equações de predição, estabelecendo uma correlação entre elas. Foram coletados 93 ejaculados que tiveram as concentrações espermáticas determinadas de forma direta (câmara de Neubauer, CN) ou indireta (espermiodensímetro, ED e espectrofotômetro, EF). Foi utilizado um delineamento em blocos casualizados, com 93 repetições e duas análises por parcela experimental. Os valores foram submetidos à análise de variância, as médias submetidas ao teste SNK e os tratamentos interrelacionados pelo ajuste do modelo linear entre os dados (equações de predição). Não houve diferença na determinação da concentração espermática obtida com ED $\left(361,7 \times 10^{6} \mathrm{sptz} / \mathrm{ml}\right)$ e EF $\left(343,7 \times 10^{6} \mathrm{sptz} / \mathrm{ml}\right)$, porém estes valores foram maiores com relação a CN $\left(245,3 \times 10^{6} \mathrm{sptz} /\right.$ $\mathrm{ml}$ ). As equações obtidas foram: $E D=-0,30$ [volume $(\mathrm{em} \mathrm{ml})]+0,61 \mathrm{EF}+213,47\left(\mathrm{R}^{2}=0,72\right)$ e $E D=-0,50$ [volume $(\mathrm{ml})]+0,47 \mathrm{CN}\left(\mathrm{R}^{2}=0,7\right)$. As técnicas de mensuração da concentração espermática pelo ED e EF superestimam os valores em relação à CN. Novos estudos devem ser conduzidos para determinar a verdadeira eficiência com que essas técnicas são empregadas a campo.

Recibido: 15-4-08. Aceptado: 8-5-08.

\section{AdDitionAl KeYWORDS}

Spectrofotometer. Spermdensimeter. Neubauer chamber.

\section{SUMMARY}

The precise and accurate determination of the spermatic concentration of the ejaculate is important in the production of insemination doses with a correct number of spermatozoa. The objective of this work was to compare the efficiency of some measurement techniques for spermatic concentration in the semen and to determine prediction equations, establishing a correlation among them. It were collected 93 ejaculations, its spermatic concentration was determined by direct (Neubauer chamber, NC) or indirect (spermdensimeter, SD and spectrophotometer, SP) methods. A randomized block design was utilized, with 93 repetitions and two analysis per experimental unit. Data were submitted to variance analysis, the SNK test was applied to means and the treatments were interrelated by means od adjustment of linear models among results (prediction equation). It was not difference in the determination of the spermatic concentration obtained with SD $\left(361.7 \times 10^{6} \mathrm{sptz} / \mathrm{ml}\right)$ and SP $\left(343.7 \times 10^{6} \mathrm{sptz} / \mathrm{ml}\right)$, but these values were higher than the NC $\left(245.3 \times 10^{6} \mathrm{sptz} / \mathrm{ml}\right)$. The equations obtained were $\mathrm{SD}=-0.30$ [ejaculate volume (in $\mathrm{ml})]+0.61 \mathrm{SP}+213.47\left(\mathrm{R}^{2}=0.72\right)$ and $\mathrm{SD}=-0.50$ [ejaculate volume (in $\mathrm{ml})]+0.47 \mathrm{NC}\left(\mathrm{R}^{2}=0.7\right)$. The $\mathrm{SD}$ and SP measurement techniques of the spermatic concentration overestimate values with regard to $\mathrm{CN}$. New research is need to determine the true efficiency of these techniques in farms.

Arch. Zootec. 59 (227): 463-466. 2010. 


\section{INTRODUÇÃO}

A inseminação artificial (IA) em suínos vem sendo cada vez mais utilizada em granjas comerciais, trazendo vantagens econômicas, tais como a diminuição na proporção de machos em relação às fêmeas e o rápido melhoramento genético do plantel.

No entanto, para o sucesso da implantação de um programa de IA, é fundamental que a qualidade do sêmen seja preservada após o processamento do ejaculado. Além das características relacionadas ao aspecto, odor e motilidade espermática, a concentração de espermatozóides é extremamente importante, não só para a adequação na produção de doses inseminantes nas centrais de inseminação, mas também para a manutenção das características de fertilidade (Hansen et al., 2002).

De acordo com Douglas-Hamilton et al. (2005), existem diversas formas de se determinar a concentração nos ejaculados, dentre elas a determinação indireta através do aspecto de densidade (densitometria) ou da absorção de luz (fotometria) e métodos diretos, como a contagem de células individuais em câmara de volume conhecido (citometria). Segundo Vianna et al. (2004), a forma direta com o uso da câmara de Neubauer é a técnica de maior confiabilidade. No entanto, não é utilizada rotineiramente em centrais de IA, uma vez que é demorada e necessita de várias mensurações de cada amostra para se obter uma boa precisão.

Com relação ao espectrofotômetro, este é o método mais freqüente nas centrais de IA (Hansen et al., 2002). Baseado no grau de absorbância de luz, permite leitura rápida e de fácil manuseio. Entretanto, segundo Andrade et al. (2001), há evidências da existência de diferenças, a partir de uma mesma amostra, em função de calibragem inadequada do aparelho.

Já o espermiodensímetro, que é baseado no grau de turbidez do ejaculado, apresenta vantagens em relação às técnicas citadas, dentre elas um menor custo, maior praticidade, rapidez e precisão. No entanto, esta técnica tende a superestimar a concentração em relação à câmara de Neubauer, levando à produção de doses menos concentradas, tendo, como conseqüência, problemas de fertilização (Vianna et al., 2004).

A eficácia com que a concentração espermática é mensurada através de diferentes metodologias, deve ser revista para assegurar bons índices reprodutivos em um plantel suinícola. Dessa forma, o objetivo foi comparar a eficiência de três técnicas de mensuração da concentração espermática do ejaculado suíno e determinar equações de predição entre as diferentes metodologias, estabelecendo uma correlação entre elas, permitindo uma melhor avaliação quantitativa do sêmen.

\section{MATERIAL E MÉTODOS}

O experimento foi realizado na granja comercial de ciclo completo pertencente à Fazenda São Paulo, município de Oliveira/ MG. Foram coletados 93 ejaculados de machos de linhagem AGROCERES PIC, pela técnica da mão enluvada, em frasco graduado, pré-aquecido a $35^{\circ} \mathrm{C}$, e protegido por recipiente isotérmico.

Após a coleta, o sêmen foi levado ao laboratório, onde foram inicialmente avaliados o volume do ejaculado e a motilidade espermática. Em seguida, foi determinada a concentração através das seguintes técnicas: câmara de Neubauer, espermiodensímetro de Karras e espectrofotômetro.

Para mensuração da concentração espermática nas três técnicas foi utilizada a metodologia descrita por Bortolozzo et al. (2005), seguindo a tabela de valores padronizados pelo fabricante.

As doses inseminantes foram calculadas considerando-se a avaliação da concentração em cada uma das técnicas, utilizando-se 4 bilhões de espermatozóides por dose. As análises estatísticas foram realizadas no programa SAS (1996), sendo determinadas equações de predição entre as técnicas utili-

Archivos de zootecnia vol. 59, núm. 227, p. 464. 
zadas e as médias de cada tratamento comparadas pelo teste SNK a 5\% de probabilidade.

\section{RESULTADOSEDISCUSSÃO}

Os valores médios encontrados para a concentração espermática, desvio padrão e número médio de doses inseminantes obtidos em cada técnica, estão apresentados na tabela I. Os resultados mostram que as concentrações obtidas através do espermiodensímetro e espectrofotômetro foram maiores $(p<0,01)$ em relação à câmara de Neubauer, e que estas não diferiram entre si.

Pela essencialidade de cada técnica, fundamentada em formas diretas e indiretas, segundo Douglas-Hamilton et al. (2005), estes resultados já eram esperados. Pelo fato de a concentração espermática ser baseada na densidade da amostra, o espermiodensímetro e o espectrofotômetro podem superestimar os resultados de concentração devido à presença, além dos espermatozóides, de células de descamação, leucócitos e sujidades que podem afetar a turbidez do ejaculado.

Segundo Hansen et al. (2002), as avaliações imprecisas da concentração de espermatozóides no sêmen in natura implicam em produção de doses que podem ter quantidade inadequada de gametas para máxima fertilidade, além do que, algumas doses contendo um número excessivo indicam uso ineficiente de machos de alto valor genético.

Com relação à acurácia dos dados, os resultados mostram, em valores numéricos, uma menor variabilidade quando se utilizou o espectrofotômetro. Hansen et al. (2002), utilizando hemocitometria e espectrofotometria na espécie suína, obtiveram coeficientes de variação, dentro de cada técnica, de 10,2 e $6,3 \%$, respectivamente, valores estes muito inferiores aos encontrados no presente experimento, que foram de 31,8; 30,2 e 52,8\% para espermiodensímetro, espectrofotômetro e câmara de Neubauer. Apesar dos autores concluírem que méto-
Tabela I. Concentração espermática média de ejaculados de suínos determinados através do espermiodensímetro, espectrofotômetro e câmara de Neubauer e número médio de doses inseminantes. (Average spermatozoa concentration in swine ejaculates determined through spermdensimeter, spectrofotometer and Neubauer chamber and average number of insemination doses).

\begin{tabular}{lcccc}
\hline & $\begin{array}{r}\text { Espermio- } \\
\text { densímetro }\end{array}$ & $\begin{array}{c}\text { Espectro- } \\
\text { fotômetro }\end{array}$ & $\begin{array}{c}\text { Câmara } \\
\text { Neubauer }\end{array}$ & $\mathrm{p}$ \\
\hline $\mathrm{N}$ & 93 & 93 & 93 & \\
$\mathrm{CM}$ & $361,67^{\mathrm{a}}$ & $343,68^{\mathrm{a}}$ & $245,31^{\mathrm{b}}$ & $<0,01$ \\
$\mathrm{DP}$ & 114,93 & 103,70 & 129,48 & \\
$\mathrm{NMD}$ & $27,12^{\mathrm{a}}$ & $25,78^{\mathrm{a}}$ & $18,40^{\mathrm{b}}$ & $<0,01$
\end{tabular}

CM: Concentração média $\left(\times 10^{6} \mathrm{sptz} / \mathrm{ml}\right)$; DP: Desviopadrão; NMD: Número médio de doses (concentração: 4 bilhões de sptz por dose).

dos baseados em densidade óptica serem considerados mais confiáveis e eficientes, deve-se levar em consideração a superestimação dos dados quando se utiliza estas técnicas. Estes resultados ressaltam a importância de se realizar várias análises a partir de um ejaculado, principalmente quando se utiliza a forma direta de contagem de espermatozóides, que, segundo Hansen et al. (2002), é a técnica que apresenta menor precisão, apesar da maior confiabilidade.

As equações obtidas a partir do estudo das interrelações entre os dados obtidos, e que permitem a verificação da confiabilidade de cada técnica utilizada, as que mais se ajustaram aos dados $(\mathrm{p}<0,01)$ foram:

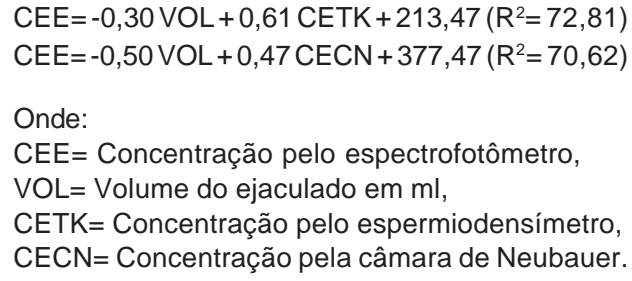

Vianna et al. (2004) obtiveram uma correlação de escala entre o espermio-den- 
símetro de Karras e a câmara de Neubauer e de acordo com esses autores, a verificação entre os valores obtidos através destas equações permite a avaliação da técnica de mensuração da concentração espermática a partir dos ejaculados, diminuindo, dessa forma, a subjetividade dos dados.

Aos valores encontrados para número de doses produzidas foram superiores $(\mathrm{p}<0,01)$ para o espermiodensímetro e espectrofotômetro em relação à câmara de Neubauer. Neste caso, as concentrações das doses inseminantes podem estar abaixo das condições especificadas, que é de 3 a 5 bilhões de espermatozóides por dose inseminante em 80 a $100 \mathrm{ml}$, segundo Bortolozzo et al. (2005). Dessa forma, o método utilizado para determinação da concentração espermática é um ponto importante para a diminuição do desperdício de células durante a preparação das doses inseminantes.

Os métodos empíricos de determinação da concentração pelo aspecto do ejaculado

\section{BIBLIOGRAFIA}

Andrade, M.C.C., Möller, G., Souza, A.M., Garbade, P. e Mattos, R.C. 2001. Comparação entre a fotometria e a hemocitometria para a determinação da concentração espermática do sêmen eqüino. Em: Salão de Iniciação Científica de Ciências Agrárias da UFRGS, XIII. Rio Grande do Sul, Brasil. Anais... Universidade Federal do Rio Grande do Sul. Rio Grande do Sul. 132 pp.

Bortolozzo, F.P., Wents, I. e Dallanora, D. 2005. Situação atual da inseminação artificial em suínos. Acta Scient. Vet., 33: 17-32.

Douglas-Hamilton, D.H., Smith, N.G., Kuster, C.E., Vermeiden, J.P.W. and Althouse, G.C. 2005. Particle distribution in low-volume capillary- são extremamente imprecisos e improdutivos, já que, tanto a subestimação quanto a superestimação, são indesejáveis. A utilização das técnicas irá depender da eficácia com que cada uma delas estima a verdadeira concentração dos ejaculados obtidos e das doses inseminantes utilizadas em programas de IA.

\section{CONCLUSÃO}

As técnicas de mensuração da concentração espermática do sêmen suíno baseadas na densidade superestimaram, em proporções semelhantes, os valores em relação à câmara de Neubauer. Novos estudos devem ser conduzidos para determinar a verdadeira eficiência com que essas técnicas são empregadas a campo.

\section{AGRADECIMENTOS}

À Fazenda São Paulo pela utilização dos reprodutores e da infra-estrutura da granja.

loaded chambers. J. Androl., 26: 107-114

Hansen, C., Christensen, P., Stryhn, H., Hedeboe, A.M., Rode, M. and Boe-Hansen, G. 2002. Validation of the FACSCount AF System for determination of sperm concentration in boar semen. Repr. Domest. Anim., 37: 330-334.

SAS. 1996. System for Windows 6.1. SAS Institute Inc. Cary. NC. USA.

Vianna, W.L., Bruno, D.G., Namindome, A., Rosseto, A.C., Rodrigues, P.H.M., Pinese, M.E. e Moretti, A.S. 2004. Estudo comparativo da eficiência de diferentes técnicas de mensuração da concentração espermática em suínos. Rev. Bras. Zootecn., 33: 2054-2059.

Archivos de zootecnia vol. 59, núm. 227, p. 466. 